\title{
Mittheilungen aus dem chemischen Laboratorium der Universität Genf.
}

\author{
Ueber Oxyketonfarbstoffe; \\ von $C$. Graebe und A. Eichengrïn.
}

(Eingelaufen den 19. Januar 1892.)

In einer kurzen Mittheilung*) haben wir vor einiger Zeit darauf hingewiesen, dass eine Carbonylgruppe schon im Stande ist, das Gefärbtsein aromatischer Verbindungen zu bedingen. Bei den Ketonen selbst tritt diese Eigenschaft nur dann hervor, wenn gleichzeitig eine stärkere Kohlenstoffcondensation vorliegt, wie bei dem deutlich gelb gefärbten Diphenylenketon, dem goldgelben Retenketon und auch schon bei dem Benzylidenacetophenon, weiches hellgelb ist.

Wird in die farblosen oder vielleicht nur ausserordentlich schwach gefärbten Ketone, wie Benzophenon oder Phenylnaphtylketon, Hydroxyl eingeführt, so tritt die Färbung stärker hervor und bei den Salzen ist sie noch intensiver, wie dies auch bei dem Tetramethyldiamidobenzophenon der Fall ist, welches schon in freiem Zustand nach der Ansicht des Einen von uns als schwach gelb gefärbt anzusehen ist $\%$ \%).

Die Gewinnung der Oxyketone durch Condensation von Säuren einerseits und ein- oder mehratomigen Phenolen andrerseits ist in theoretischer Beziehung auf eine Arbeit von

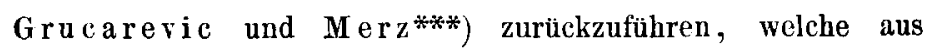
Benzoylchlorid, Phenol und Zink das Benzoat des p-Oxybenzophenons erhielten. Das Zink wirkt hier wohl in Form vor Zinkchlorid und in der That haben Doebner und Stackmann es später mit Vortheil durch letzteres ersetzt, nachdem sie

*) Ber. d. deutsch. chem. Ges. 24, 967 .

**) Ber. d. deutsch. chem. Ges. 20, 3263.

***) Ber. d. deutsch, chem. Ges. 6, 1245. (1873.) 
vorher schon gezeigt hatten, dass man an Stelle ron Benzoy]chlorid auch das Benzotrichlorid*) verwenden kann.

Dass die Oxyketone direct aus Säuren und Phenolen erhalten werden können, wurde dann gleichzeitig ron Arthur Michael**) und von Nencki und Sieber***) aufgefunden, Der erstere bewirkte die Condensation aromatischer Oxysäuren mit Phenolen durch Zinnchlorid oder Chlorzink. Nencki und Sieber erhielten aus Eisessig, Resorcin und Zinkchlorid ein Dioxyacetophenon, und aus Pyrogallol, Eisessig mit demselben wasserentziehenden Mittel das Galloacetophenon.

René Bohn hat zuerst (1889) die Bedeutung der aromatischen Oxyketone als Farbstoffe erkannt und darauf hingewiesen, dass dieselbe durch das Vorhandensein von mindestens zwei benachbarten Hydroxylen bedingt ist. Durch Condensation fetter oder aromatischer Carbonsäuren mit Pyrrogallol und von Gallussäure oder Pyrogallolcarbonsäure mit ein- oder mehrwerthigen Phenolen erhielt er eine grosse Zahl von Oxyketonen, weiche die Eigenschaft besitzen, gebeizte Baumwolle in ähnlicher Weise wie Alizarin zu färben und gelbe oder grünlichgelbe Lacke zu bilden. Von den fünfzchn in den Patenten der Badischen Anilin- und Sodafabrik $\dagger$ ) aufgefülırten Farbstoffen wird augenblicklich nur das aus Benzoësäure oder Benzotrichlorid und Pyrogallol crhaltene Trioxybenzophenon unter dem Namen Alizaringelb $A$ in den Handel gebracht $\uparrow \dagger$ ). Ausser demselben laben wir noch den aus Salicylsäure und Phenol, sowie den aus Gallussäure und $\alpha$-Naphtol entstehenden

*) Ber. d. dentsch. chem. Ges. 9, 1919. (1876)

**) Am. Chem. Journ, 5, 83. (18\$1.)

***) Journ. f. prakt. Chemie 23, 147. (1881.)

†) D. Reichspatente Nr. 49149 und 50450 (1889); Ber. d. deutsch. chem. Ges. 23 c. 43 , und 188.

††) Ferner wird von derselben Fabrik das Galloacetophenon fabricirt und in der Industrie als Alizaringelb $\mathrm{C}$ bezeichnet. Es liefert Lacke, die bedeutend grünlicher sind als die des Trioxybenzophenons. 
Farbstoff, die wir beide der Liebenswürdigkeit von Herrn Dr. Bohn verdanken, untersucht.

Unsere Beobachtungen ergeben durchweg die Richtigkeit der von Bohn aufgestellten Formeln. Es hat sich allgemein gezeigt, dass in diese Oxyketone sich ebensovielmal Acetyl einführen lässt, als Hydroxyle vorhanden sind; dass sich dagegen nicht oder nur sehr schwierig alle Hydroxylwasserstoffe durch Methyl oder Aethyl ersetzen lassen. In Betreff der Constitution war es nun bisher nicht möglich, zwischen den beiden, der Synthese entsprechenden Formeln des Trioxybenzophenons zu entscheiden, dagegen konnten wir für das Tetraoxybenzophenon die Stellung der Hydroxyle sicher ermitteln und für das Tetraoxynaphtylphenylketon mit grosser Wahrscheinlichkeit herleiten.

Um die Constitution der betreffenden Oxyketone angeben zu können, schlagen wir folgende Schema vor. Wir glauben, dass dieselben zweckmässig zur Bezeichnung aller Derivate der aromatischen Ketone benutzt werden können.
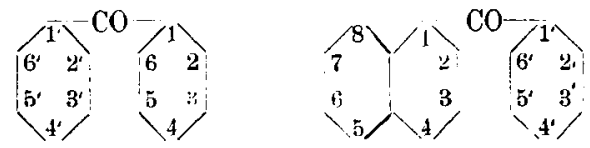

I Trioxybenzophenon 2, 3, 4 oder 3, 4, 5. (Alizaringelb A.)

Dieses Trioxybenzophenon wurde, wie in dem Hauptpatent der Badischen Anilin- und Sodafabrik genau angegeben ist, znerst durch Erhitzen von Benzoësäure, Pyrogallol und Chlorzink auf $145^{\circ}$ gewounen. An Stelle von Benzoësäure können Benzoylchlorid, Benzoësäureanhydrid ${ }^{*}$ ) und Benzotrichlorid **) benutzt werden. Technisch wird jetzt das Alizaringelb A durch Einwirkung von Benzotrichlorid auf Pyrogallol dargestellt. Dasselbe findet in der Kattundruckerei Anwendung, wo es zur Herstellung

*) Deutsches Reichspatent Nr. 504ð̌1 (1889).

**) Deutsches Reichspatent Nr. 54661 (1890); Ber. d. deutsch. chem. (irs. 24, 3rs c. 
ecbter reingelber Nüancen gebraucht wird. Am zweckmässigsten wird es genau wie Alizarinroth mit ciner Mischung von Thonerde- und Kalkbeize aufgedruckt oder gefärbt. Seine Lacke sind goldgelb und gegen Licht, Iuft, Seife etc. sehr widerstandsfähig.

Zu unserer Untersuchung haben wir theils ein schön krystallisirtes Product, theils die Handelswaare, welche wir der Badischen Anilin- und Sodafabrik rerdanken, benutzt. Die wesentlichsten Eigenschaften des Farbstoffs sind schon im Patent genau beschrieben. Aus dem käuflichen Alizaringelb A. (Paste von $20 \mathrm{pC}$. Farbstoffgehalt) erhält man das reine Trioxybenzophenon am besten durch Krystallisiren aus verdünntem Alkohol.

In Betreff des Schmelzpunktes, der im Patent bei $137-138^{\circ}$ angegeben ist, muss der Krystallwassergehalt mit in Betracht gezogen werden. Erwärmt man die Substanz allmählich von einer unter $100^{\circ}$ liegenden Temperatur an, so beobachtet man das Sehmelzen erst bei $140-141^{\circ}$ (corr.). Bringt man aber das Schmelzpunktsröhrchen in das schon bis etwas über $100^{\circ}$ am besten auf $110^{\circ}$ erwärmte Bad, so tritt ein Schmelzen ein, dann erstart die Substanz wieder und zeigt darauf den Schmelzpunkt $140-141^{\circ}$ der wasserfreien Substanz. Unter Wasser schmilzt das Trioxybenzophenon zu einem gelben Oel. Das Krystallwasser entweicht leicht und vollständig bei $100^{\circ}$.

$0,2940 \mathrm{~g}$ verloren $0,0220 \mathrm{H}_{\mathbf{2}} \mathrm{O}$.

$$
\begin{array}{ccc} 
& \text { Berechnet für } & \text { Gefunden } \\
& \mathrm{C}_{13} \mathrm{H}_{10} \mathrm{O}_{4}+\mathrm{H}_{2} \mathrm{O} & \\
\mathrm{H}_{2} \mathrm{O} & \boldsymbol{i}, 26 & \boldsymbol{i}, 4 \mathrm{~s}
\end{array}
$$

$0,1605 \mathrm{~g}$ bei $100^{\circ}$ getrockneter Substanz gaben $0,3980 \mathrm{CO}$, und $0,0635 \mathrm{H}_{2} \mathrm{O}$.

$\begin{array}{ccc} & \text { Berechnet für } & \text { Gefunden } \\ & \mathrm{C}_{13} \mathrm{H}_{10} \mathrm{O}_{4} & \\ \mathrm{C} & 67,82 & 67,62 \\ \mathrm{H} & 4,35 & 4,3 \mathrm{~S}\end{array}$

Das 'Trioxybenzophenon krystallisirt in gelben Nadeln; in fein vertheiltem Zustand ist die Farbe heller und die käufliche Paste hat eine graugelbe Färbung. Der Farbstoff löst sich 
Graebe uni Eichengrün, Oxyketonfarbstoffe. $\quad 299$

schwer in kaltem, reichlicher in heissem Wasser, wenig in Benzol und leicht in Alkohol, Aether, Aceton und Eisessig. In concentrirter Schwefelsäure löst er sich mit intensiv gelber Farbe. In gleicher Weise sind seine Lösungen in Alkalien gefärbt. In überschüssiger Natronlauge gelöst, zieht er Sauerstoff aus der Luft an und es wird ein grünes Oxydationsproduct gebildet. Aus den Lösungen in Carbonaten wird es unverändert gefällt und zwar schon durch Kohlensäure.

Aus dem chemischen Verhalten war es nicht möglich zu ermitteln, welche von den beiden aus der Bildungsweise sich berleitenden Formeln, 2, 3, 4 oder 3, 4, 5,

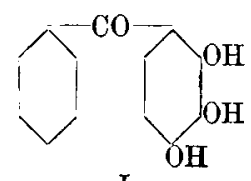

I.

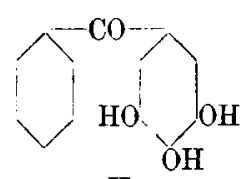

II.

die richtige ist. Für die erstere spricht immerhin die Thatsache, dass bei der Bildung des Tetraoxybenzophenons aus Salicylsäure und Pyrogallol der Eintritt des Carbonyls so erfolgt, dass die Hydroxyle die Stellung 2, 3, 4 einnehmen. Wir haben versucht, diese Frage durch Condensation von Gallussäure mit Benzol zu entscheiden, doch ist es uns nicht geglückt, auf diese Weise zu einem Trioxybenzophenon zu gelangen.

\section{Salze und Aether des Trioxybenzophenons.}

Das Natriumsalz, $\mathrm{C}_{13} \mathrm{H}_{9} \mathrm{O}_{4} \cdot \mathrm{Na}$, erbält man am einfachsten, wenn man das Trioxybenzophenon in Wasser suspendirt und mit der berechneten Menge Natriumcarbonat, oder auch einem Ueberschuss des letzteren erwärmt. Aus Wasser, in dem es schwer löslich ist, umkrystallisirt, bildet es schön gelbe Nadeln, welche kein Krystallwasser enthalten.

0,1235 g gaben $0,0350 \mathrm{SO}_{4} \mathrm{Na}_{2}$.

$\begin{array}{ccc} & \text { Berechnet } & \text { Gefunden } \\ \mathrm{Na} & 9,13 & 9,20\end{array}$


Das Kaliumsalz, $\mathrm{C}_{13} \mathrm{H}_{9} \mathrm{O}_{4} \mathrm{~K}$, kann in derselben Weise erhalten werden, wie das Natriumsalz. Wir haben es auch so dargestellt, dass unter Luftabschluss alkoholisches Kali zu einer alkoholischen Lösung des Trioxybenzophenons zugegeben wurde. Es scheidet sich sofort ein hellgelbes Pulver aus, welches kein Krystallwasser enthält und aus dem Monokaliumsalz besteht.

Lässt man dagegen die Trioxybenzophenonlösung in alkoholisches Kalihydrat fliessen, so entsteht kein Niederschlag, sondern eine blutrothe Lösung, aus der sich durch Aether ein kaliumreicheres Salz fällen lässt; doch wurde es nicht in reinem Zustand erhalten.

Lin Bleisalz, $\mathrm{C}_{13} \mathrm{H}_{\mathrm{s}} \mathrm{O}_{4} \mathrm{~Pb}$, welches sich vom Trioxybenzophenon durch Ersatz von zwei Atomen Wasserstoff herleitet, erhält man durch Fällen einer ammoniakalischen Lösung des Ketons mit Bleiacetat. Es besteht aus einem hellgelben, in Wasser unlöslichen Pulver.

$0,2700 \mathrm{~g}$ gaben $0,1870 \mathrm{SO}{ }_{4} \mathrm{~Pb}$.

$\begin{array}{ccc} & \text { Berechnet } & \text { Gefunden } \\ \mathrm{Pb} & 4 \bar{\imath}, 4 \check{5} & \mathbf{4 7 , 3 0}\end{array}$

Acetyllerirat, $\mathrm{C}_{13} \mathrm{H}_{7} \mathrm{O}_{4}\left(\mathrm{COCH}_{3}\right)_{33}$. Das schon im Patent angeführte Acetylderivat, welches aus weissen Blättchen besteht, die bei $117^{\circ}$ schmelzen, haben wir durch Kochen des Trioxybenzophenons mit Essigsäureashydrid während ungefähr drei Stunden dargestellt. Dasselbe ist in Alkohol, Eisessig und Benzol löslich, dagegen in Wasser, Ligroïn und Aether unlöslich. Aus der Analyse geht hervor, dass sich eine triacetylirte Verbindung gebildet hat.

$0,1800 \mathrm{~g}$ gaben $0,4220 \mathrm{CO}_{2}$ und $0,0750 \mathrm{H}_{8} \mathrm{O}$.

$\begin{array}{ccc} & \text { Berechnet } & \text { Gefunden } \\ \mathrm{C} & 64,03 & \mathbf{6 3 , 9 1} \\ \mathrm{H} & \mathbf{4 , 4 9} & \mathbf{4 , 6 2}\end{array}$

Methyläther. Bei den Versuchen, das Trioxybenzophenon zu methyliren, zeigte es sich, dass sich leicht ein Methyl einführen lässt, dass, um den Dimethyläther zu erhalten, besondere 
Graebe und Eichengriin, Oxylietonfarbstoffe. 301

Versuchsbedingungen einzuhalten sind und dass der Trimethyläther sich nicht oder nur äusserst schwierig bildet. Bemerkenswerth ist es, dass der Monomethyläther gelb und in Alkalien löslich ist, während der Dimethyläther farblos erhalten werden kann und sich in Natronlauge nur sehr schwierig löst. Es erinnert dies Verhalten an die Beobachtungen von J. Herzig über Euxanthon*).

Der Monomethyläther, $\mathrm{C}_{13} \mathrm{H}_{9} \mathrm{O}_{4} \cdot \mathrm{CH}_{3}$, bildete sich als Hauptproduct beim Erwärmen des Trioxybenzophenons mit 3 Mol. Kalihydrat, etwas mehr wie $3 \mathrm{Mol}$. Jodmethyl und Methylalkohol während einiger Stunden auf $100^{\circ}$. Bis auf geringe Mengen einer öligen Substanz war das erhaltene Product in Natronlauge in der Kälte leicht löslich. Der aus der alkalischen Lösung durch Salzsäure ausgefällte Aether wurde unter Zusatz von etwas Thierkohle aus Alkohol krystallisirt und in Form schwefelgelber Krystalle erhalten, welche bei $165^{\circ}$ schmelzen und die $\mathrm{Zu}$ sammensetzung des Monomethyläthers besitzen.

$$
\begin{array}{ccc}
0,1470 \mathrm{~g} \text { gaben } & 0,36 \times 0 \mathrm{CO} . \text { und } & 0,0620 \mathrm{H}_{2} \mathrm{O} . \\
& \text { Berechnet } & \text { Gefunde } \\
\mathrm{C} & 68,84 & 65, \check{0} 6 \\
\mathrm{H} & 4,92 & 5,06
\end{array}
$$

Am besten lässt sich aber dieser Aether durch Erwärmen des Mononatriumsalzes des Trioxybenzophenons mit etwas überschüssigem Jodmethyl während einiger Stunden auf $120^{\circ}$ darstellen. Der Monomethyläther löst sich leicht in Chloroform, schwerer in Alkohol, Aether und Eisessig und sehr wenig in Benzol. Er löst sich in der Kälte in verdünnter Natronlauge, dagegen nur sehr langsam in Alkalicarbonaten. Erstere liefert dunkelgelbe und letztere hellgelbe Lösungen.

$\mathrm{Da}$ aus dem Verhalten des Trioxybenzophenons gegen Natriumcarbonat hervorgeht, dass ein Hydroxyl ziemlich stark saure Eigenschaften besitzt, so wurde versucht, direct den

*) Monatshefte für Chemie 12, 18 ;. 
Monometbyläther durch Erhitzen des Trioxybenzophenons mit Methylalkohol und Salzsäure darzustellen, ohne dass aber eine Methylirung erzielt wurde.

Der Dimethyläther, $\mathrm{C}_{13} \mathrm{H}_{8} \mathrm{O}_{4}\left(\mathrm{CH}_{3}\right)_{2}$, wurde an besten erhalten durch 6 stündiges Erwärmen von 2,5 g des Mononatriumsalzes vom Trioxybenzophenon, $1 \mathrm{~g} \mathrm{Na}_{2} \mathrm{CO}_{3}$ und $5 \mathrm{~g}$ Jodmethyl auf $160^{\circ}$. Man destillirt das überschüssige Jodmethyl ab und versetzt mit Wasser. Es scheidet sich ein bald krystallinisch erstarrendes Oel aus, welches man auf Thontellern trocknet. Zur Reinigung löst man es in Chloroform, fügt ein wenig Ligroin zu, wodurch harzige Verumreinigungen gefällt werden, von denen man die Flüssigkeit abgiesst, und versetzt dann mit einer grösseren Menge Ligroïn. Es scheiden sich bräunliche Krystalle aus, die durch Umkrystallisiren aus Alkohol unter Zusatz von Thierkohle farblos erhalten werden können. Sie sind in Alkohol, Chloroform und Aether leicht löslich und schmelzen bei $131^{\circ}$.

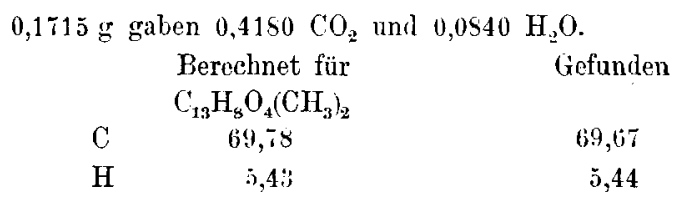

Der Dimethyläther bildet sich auch, aber weniger reichlich, durch Erhitzen des oben erwähnten Bleisalzes mit Jodmethyl auf $160^{\circ}$. Der Dimethyläther ist in Natriumcarbonat ganz unlöslich und geht auch bei anhaltendem Schütteln mit verdünnter Natronlauge nur sehr schwierig und nur theilweise in Lösung. In warmer Natronlange löst er sich mit gelber Farbe. Dass derselbe aber noch ein Hydroxyl enthält, geht aus dem Verhalten gegen Essigsäureanhydrid hervor, mit welchem er während zweier Stunden bis zum schwachen Sieden erhitzt wurde.

Es wurde so ein Acetyldimethyltrioxybenzophenon, $\mathrm{C}_{13} \mathrm{H}_{7} \mathrm{O}\left(\mathrm{OCH}_{3}\right)_{2}\left(\mathrm{O}_{0} \mathrm{CH}_{3} \mathrm{CO}\right)$ erhalten, welches sich aus Eisessig gut krystallisiren lässt und aus farblosen Prismen von $98^{\circ}$ Schmelzpunkt besteht. 
Graebe und Eichengrün, Oxyletonfarbstoffe. 303 $0,1575 \mathrm{~g}$ gaben $0,3940 \mathrm{CO}_{2}$ und $0,0750 \mathrm{H}_{2} \mathrm{O}$.

$\begin{array}{ccc} & \text { Berechnet } & \text { Gefunden } \\ \mathrm{C} & \mathbf{6 8 , 0 0} & \mathbf{6 8 , 2 0} \\ \mathrm{H} & \mathbf{\mathbf { 5 } , 3 4} & \overline{\mathbf{5}}, \mathbf{3 0}\end{array}$

Versuche, den Dimethyläther nochmals zu methyliren und so zu dem Trimethyttrioxybenzophenon zu gelangen gaben wesentlich ein negatives Resultat. Als Dimethyläther mit $1 \mathrm{Mol}$. Kalihydrat, Methyljodid und Methylalkohol während 6 Stunden auf $150^{\circ}$ erhitzt worden war, wurde er fast vollständig unverändert wieder erhalten und es hatte sich nur eine sehr geringe Menge eines in warmer Natronlauge unlöslichen Oels gebildet, aus dem nur Spuren einer krystallisirten, farblosen Substanz von $124^{0}$ Schmelzpunkt erhalten wurden. Auch bei längerem oder höherem Erhitzen konnten diese Krystalle nicht in grösserer Menge erhalten werden, obwohl sich das in warmer Natronlauge unlösliche Oel etwas reichlicher gebildet hatte.

\section{Hydrazon und Acetoxim.}

Durch Erwärmen des Trioxybenzophenons mit Phenylhydrazin in alkoholischer Lösung wurde ein harziges Reactionsproduct gebildet, aus dem sich kein krystallisirter Körper isoliren liess; aber aus dem Triacetyl-Trioxybenzophenon entstand durch Erhitzen mit Phenylhydrazin und Eisessig eine farblose, in Blättchen krystallisirende Verbindung, die bei $130^{\circ}$ schmilzt.

$0,1050 \mathrm{~g}$ gaben $5,8 \mathrm{ccm}$ Stickgas bei $14^{\circ}$ und $724 \mathrm{~mm}$ Druck.

$$
\begin{array}{ccc} 
& \text { Berechnet für } & \text { Gefunden } \\
& \mathrm{C}_{6} \mathrm{H}_{2}(\mathrm{O} . \mathrm{COCH})_{3} & \\
& \mathrm{C}_{6} \mathrm{H}_{5}>\mathrm{C}=\mathrm{N}-\mathrm{NH} \cdot \mathrm{C}_{6} \mathrm{H}_{5} & \\
\mathrm{~N} & 6,2 \mathrm{~S} & 6,60
\end{array}
$$

Auch bei Einwirkung von Hydroxylamin auf Trioxybenzophenon wurde ein harziges Product gebildet, welches sich nicht in krystallisirten Zustand überführen liess. Bessere Resultate gab gleichfalls der Acetyläther, welcher in alkoholischer Lösung mit Hydroxylaminchlorhydrat und der genau letzterem entsprechenden Menge Natriumcarbonat während etwa 15 Stunden 
zum Sieden erhitzt wurde. Man erhält anfangs eine harzige Masse, aus der durch Kochen mit Thierkohle und Alkohol sich gelbliche Xädelchen isoliren liessen, welche bei $135^{\circ}$ schmelzen und in Alkohol, Aether und Chloroform gut löslich sind.

$0,1200 \mathrm{~g}$ gaben $4 \mathrm{ccm}$ Stickgas bei $14^{\circ}$ und $724 \mathrm{~mm}$ Druck.

$$
\begin{aligned}
& \text { Berechnet für Gefunden } \\
& \mathrm{C}_{6} \mathrm{H}_{2}\left(\mathrm{O} . \mathrm{COCH}_{3}\right)_{3} \\
& \mathrm{C}_{6} \mathrm{H}_{5}> \\
& \mathrm{N} \quad 3, \pi i \quad 4,111
\end{aligned}
$$

\section{Verhalten des Trioxybenzophenons.}

Das Verhalten gegen verschiedene Reagentien wurde wesentlich in der Absicht untersucht, um die relative Stellung des Carbonyls zu den Hydroxylen zu ermittelu. So wurde versucht, mittelst Phosphorchtorid die Hydroxyle durch Chlor zu ersetzen, um durch Oxydation den andern Benzolkern zu zerstören und so zu einer Trichlorbenzoësäure zu gelangen, wodurch die Constitutionsfrage sich hätte entscheiden lassen. Es wurde aber durch Erwärmen von Trioxybenzophenon mit 3 Molekülen Fünffachchlorphosphor wesentlich ein phosphorhaltiger Körper erhalten. Derselbe entstand ausschliesslich bei Einwirkung des Phosphorchlorids auf in Toluol gelöstes Trioxybenzophenon. Lässt man nach mehrstïndigem Erlitzen die dunkclroth gefärbte Lösung abkühlen, so scheiden sich braunrothe Krystalle aus, die in Wasser, Allohol, Aether und Eisessig löslich und in Toluol sowie Ligroïn unlöslich sind. Der Phosphorbestimmung nach könnte er als ein Aether der Phosphorsäure angesehen werden und vielleicht der Formel $\mathrm{C}_{6} \mathrm{H}_{5} \cdot \mathrm{CO} . \mathrm{C}_{6} \mathrm{H}_{2} \mathrm{O}_{3}(\mathrm{PO})$ entsprechen, welche $11,31 \mathrm{pC} . \mathrm{P}$, verlangt während 11,68 gefunden wurden.

Bei der Reduction des Trioxybenzophenons mit Jodwasserstoff und Phosphor wurde eine phosphorhaltige Substanz, dagegen beim Erhitzen mit Zinkstaub in sehr geringer Menge ein Körper erhalten, welcher den Eigenschaften nach Diphenylmethan ist. 
Durch Einwirkung von Oxydationsmitteln wird, wie zu erwarten war, das hydroxylirte Benzol zerstört und Benzoësäure gebildet. Durch Permanganat erfolgt die Oxydation in alkalischer Lösung bei gewöhnlicher Temperatur. In gleicher Weise wirkt Kalihydrat, wenn man so stark erhitzt, dass die ursprünglich gelbe Schmelze farblos wird. Aus der Schmelze liess sich nur Benzoësäure extrahiren.

In concentrirter Schwefelsäure löst sich das Trioxybenzophenon in der Kälte mit rother Farbe, welche beim Erwärmen auf dem Wasserbad in eine braune übergeht. Es bildet sich in theoretischer Menge Benzoësäure, die man nach dem Verdünen mit Wasser mit Aether ausziehen kann, und in Lösung bleibt eine Sulfonsäure, welche mit Barytwasser die violette Reaction der Pyrogallosulfonsäure liefert*).

Salpetersäure wirkt entweder oxydirend oder nitrirend; es wurden Benzoësäure, Mono-, Di- und Trinitrotrioxybenzophenon erhalten. Die Umwandlung in Benzoësäure tritt als Hauptreaction auf, wenn man Trioxybenzophenou in Wasser suspendirt und tropfenweise concentrirte Salpetersäure hinzugiebt, bis eine Reaction beginnt.

Erwärmt man Trioxybenzophenon vorsichtig mit verdünter Salpetersäure $(20 \mathrm{pC}$.) auf dem Wasserbad und mässigt die Reaction, wenn sie zu heftig wird, durch Abkühlen, so bilden sich Mononitrotrioxybenzophenon, Benzoësäure und ein mit Wasserdämpfen flüchtiger Nitrokörper. Das beim Erkalten sich ausscheidende Product wurde mit Wasserdämpfen der Destillation unterworfen. Der Rückstand aus heissem Wasser krystallisirt lieferte einen bei $123^{\circ}$ schmelzendeu, gelblich gefärbten Körper. Derselbe ist in heissem Wasser, Alkohol und Aether löslich.

Die Analysen ergaben Zahlen, die einem Mononitroderivat entsprechen.

*) H. Schiff. Diese Annalen 178, 181. 


$\begin{array}{cccc} & 0,1380 \mathrm{~g} \text { gaben } & 0,2850 \mathrm{CO}_{2} & \text { und } 0,0490 \mathrm{H}_{2} \mathrm{O} . \\ & \text { Berechnet für } & \text { Gefunden } \\ & \mathrm{C}_{13} \mathrm{H}_{9} \mathrm{O}_{4} \cdot \mathrm{NO}_{2} & \\ \mathrm{C} & 56,73 & 56,30 \\ \mathrm{H} & 3,27 & 3,94\end{array}$

In Alkalien löst er sich mit gelber Farbe. Chamäleon in alkalischer Lösung verwandelt ihn in Benzoësäure; die Nitrogruppe ist also in das hydroxylirte Benzol eingetreten.

Trägt man vorsichtig in sehr kleinen Portionen das Trioxybenzophenon in Salpetersäure von 1,4 sp. G. ein, so löst sich der Farbstoff unter Erwärmen und nach dem Erkalten scheidet sich theils ein gelber Niederschlag, theils ein bald erstarrendes Oel aus. Aus dem erhaltenen Product liess sich ein in diesem Lösungsmittel schwer löslicher Körper isoliren, dessen Zusammensetzung einem Binitrotrioxybenzophenon entspricht.

$\begin{array}{ccc} & 0.1280 \text { g gaben } 0,2280 \mathrm{CO}_{2} \text { und } & 0,0380 \mathrm{H}_{2} \mathrm{O} . \\ & \text { Berechnet für } & \text { Gefunden } \\ & \mathrm{C}_{\mathbf{1 3}} \mathrm{H}_{\mathbf{8}} \mathrm{O}_{4}\left(\mathrm{NO}_{2}\right)_{2} & \\ \mathrm{C} & 48,75 & 48,59 \\ \mathrm{H} & 2,56 & 2,86\end{array}$

Dasselbe ist hellgelb, schmilzt unter Zersetzung bei $133^{\circ}$, löst sich in geringer Menge in Wasser und Alkohol mit intensiv gelber Farbe, leicht und mit derselben Färbung in Alkalien.

Bei stärkerer Einwirkung von concentrirter Salpetersäure wurde ein hellgelbes, bei $118^{\circ}$ schmelzendes Trinitrotrioxybenzophenon erhalten, welches in Wasser schwer, leicht in Alkohol löslich ist.

$\begin{array}{ccc} & 0,1225 \mathrm{~g} \text { gaben } 0,1920 \mathrm{CO}_{9} \text { und } & 0,0195 \mathrm{H}_{2} \mathrm{O} . \\ & \text { Berechnet für } & \text { Gefunden } \\ & \mathrm{C}_{13} \mathrm{H}_{7} \mathrm{O}_{4}\left(\mathrm{NO}_{2}\right)_{3} & \\ \text { C } & 42,71 & 42,80 \\ \mathrm{H} & 1,92 & 2,25\end{array}$

Fügt man Brom, welches mit Eisessig verdünnt ist, zu einer Lösung von Trioxybenzophenon in Cloroform oder Alkohol, so erwärmt sich die Flüssigkeit und nach einiger Zeit scheidet sich 
ein krystallinischer Körper aus. Durch Krystallisiren aus Alkohol werden citronengelbe Säulen erhalten, welche bei $149^{\circ}$ (corr.) schmelzen und aus Bromtrioxybenzophenon bestehen. Dasselbe ist in Alkohol, Aether und Eisessig ziemlich leicht, schwer in Benzol löslich.

$\begin{array}{ccc} & 0,1975 \mathrm{~g} \text { gaben } 0,3650{ }^{\circ} \mathrm{CO}_{2} \text { und } 0,0600 \mathrm{H}_{2} \mathrm{O} . \\ & 0,1410 \mathrm{~g}, \quad 0,0550 \mathrm{AgBr} . \\ & \\ & \text { Berechnet für } & \text { Gefunden } \\ & \mathrm{C}_{19} \mathrm{H}_{9} \mathrm{O}_{4} \mathrm{Br} \\ \mathrm{C} & 50,49 & 50,40 \\ \mathrm{H} & 2,91 & 3,33 \\ \mathrm{Br} & 25,89 & 25,66\end{array}$

Da es durch Kaliumpermanganat leicht zu Benzoësäure oxydirt wird, so ist das Brom in den sauerstoffhaltigen Kern eingetreten und die Formel $\mathrm{C}_{6} \mathrm{H}_{5} \cdot \mathrm{CO} \cdot \mathrm{C}_{6} \mathrm{HBr}$. $(\mathrm{OH})_{3}$ für dasselbe anzunehmen. Durch Salpetersäure wird es in vollkommen bromfreies Mononitrotrioxybenzophenon übergeführt, welches mit dem oben beschriebenen identisch ist.

II. 2, 3, 4, 2' Tetraoxybenzophenon.<smiles>O=C(O)C1CCCCC1O</smiles>

Das aus Salicylsäure und Pyrogallol erhaltene Tetraoxybenzophenon bildet grünlichgelbe Blättchen; es ist in Wasser, Benzol und Ligroin schwer, dagegen in Alkohol, Aether und Eisessig leicht löslich. Es enthält ein Molekül Krystallwasser, welches bei Wasserbadtemperatur entweicht, wobei die Krystalle ihren Glanz verlieren. Der Schmelzpunkt der wasserhaltigen Substanz liegt beim Siedepunkte des Wassers. Fährt man mit dem Erwärmen fort, so wird die Substanz wieder fest und schmilzt dann bei $149^{\circ}$ (corr.). Die wasserfreie Verbindung ist schwerer löslich als die wasserhaltige. 
$0,1680 \mathrm{~g}$ bei gewöhnlicher Temperatur getrocknet gaben $0,3680 \mathrm{CO}$ und $0,0705 \mathrm{H}_{2} \mathrm{O}$.

$0,2855 \mathrm{~g}$ verloren bei $100^{\circ} 0,0200 \mathrm{H}_{2} \mathrm{O}$.

$\begin{array}{lcr} & \text { Berechnet für } & \text { Gefunden } \\ & \mathrm{C}_{13} \mathrm{H}_{10} \mathrm{O}_{5}+\mathrm{H}_{2} \mathrm{O} & \\ \mathrm{C} & \mathbf{5 9} 9,10 & 55,90 \\ \mathrm{H} & \mathbf{4 , 5 5} & \mathbf{4 , 6 6} \\ \mathrm{H}_{2} \mathrm{O} & \mathbf{6 , 8 2} & 7,00\end{array}$

Bei vorsichtigem Erhitzen ist es sublimirbar und man erhält Nädelchen, die nur äusserst wenig gefärbt sind, wie dies auch bei dem ans alkoholischer Lösung in fein vertheiltem Zustand ausgefällten Tetraoxybenzophenon der Fall ist.

Die Salze des T'etraoxybenzophenons sind alle viel intensiver gefärbt als das Keton. Löst man dieses in Natriumcarbonat, so erhält man eine gelbe Lösung; wendet man dagegen Natronhydrat oder Aetzkali an, so ist dieselbe gelbroth gefärbt, wird aber an der Luft rasch dunkelgrün und scheidet dann einen dunkelgefärbten Niederschlag aus. Ammoniak löst das Tetraoxybenzophenon mit intensiv rothgelber Färbung. Aus den alkalischen Lösungen wird das Keton durch überschüssige Kohlensäure gefällt. Die Lösungen des Natrium- oder Ammoniumsalzes geben mit einem Thonerdesalze einen goldgelben Niederschlag, mit Blei- und Magnesiumlösungen rothgelbe, mit Chlorcalcium grünlichgelbe und mit Eisenchlorid gelbbraune Fällungen. Besonders charakteristisch ist das Mononatriumsalz. Suspendirt man das Keton in heissem Wasser und setzt entweder die auf ein Atom Natrium berechnete Menge Natriumcarbonat oder auch einen Ueberschuss davon hinzu, so tritt zuerst Lösung ein und darauf scheiden sich rasch schwefelgelbe Kryställchen aus. Dieselben sind in Wasser ziemlich schwer löslich und enthalten 1 Mol. Krystallwasser.

$0,2465 \mathrm{~g}$ gaben bei $115^{\circ}$ einen Gewichtsverlust von $0,0160 \mathrm{~g}$ und lieferten dann $0,0585 \mathrm{Na}_{2} \mathrm{SO}_{4}$.

$\begin{array}{lcc} & \text { Berechnet für } & \text { Gefunden } \\ & \mathrm{C}_{\mathbf{1 3}} \mathrm{H}_{9} \mathrm{O}_{\mathbf{5}} \mathrm{Na}+\mathrm{H}_{2} \mathrm{O} & \\ \mathrm{Na} & \mathbf{8 , 2 1} & 8,27 \\ \mathrm{H}_{2} \mathrm{O} & 6,30 & 6,50\end{array}$


Graebe und Eichengrün, Oxyketonfarbstoffe. $\quad 309$

Acetylderivat des Tetraoxybenzophenons.

Entwässertes Keton wurde drei Stunden lang mit Essigsäureanhydrid bis zum Sieden erhitzt. Beim Stehen unter Wasser verwandelte es sich nach und nach in eine spröde Masse, welche sich dann leicht aus Alkohol oder Eisessig umkrystallisiren lässt und farblose Krystallschuppen vom Schmelzpunkt $118^{\circ}$ bildet.

Die Analyse ergab Zahlen, welche einem Tetraucetylderivat entsprechen.

$\begin{array}{cccc} & 0,1565 \mathrm{~g} \text { gaben } 0,3470 \mathrm{CO}_{2} \text { und } & 0,0650 \mathrm{H}_{2} \mathrm{O} . \\ & \text { Berechnet für } & \text { Gefunden } \\ & \mathrm{C}_{\mathbf{1 3}} \mathrm{H}_{6} \mathrm{O}_{5}\left(\mathrm{C}_{\mathbf{2}} \mathrm{H}_{8} \mathrm{O}\right)_{4} & \\ \mathrm{C} & \mathbf{6 0 , 8 7} & 60,50 \\ \mathrm{H} & \mathbf{4 , 3 5} & 4,50\end{array}$

Die alkoholische Lösung dieser Acetylverbindung mit Phenylhydrazin in der Kälte versetzt liefert farblose Nadeln eines stickstoffhaltigen bei $129^{\circ}$ schmelzenden Körpers. Es hat sich also ein Hydrazon gebildet.

Verhalten des Tetraoxybenzophenons.

In concentrirter Schwefelsäure löst sich das Tetraoxybenzophenon in der Kälte mit gelbrother Farbe uud wird auf Wasserzusatz wieder unverändert gefällt. Erwärmt man aber auf $100^{\circ}$ so erfolgt Spaltung; es bildet sich Salicylsäure und eine Sulfosäure, welche die Farbenreactionen der Pyrogallolsulfonsäure zeigt. Ebenso wirkt mit dem gleichen Volum Wasser verdünnte Schwefelsäure bei $140^{\circ}$. Bei diesem Tetraoxybenzophenon erfolgt also der Uebergang in das entsprechende Xanthonderivat nicht so leicht wie bei der Euxanthonsäure, die sich unter denselben Bedingungen in Euxanthon verwandelt. Möglicherweise ist bei der Temperatur ron $100^{\circ}$ die räumliche Lagerung der beiden Benzole keine solche, dass durch Wasserabspaltung die Ringbildung erfolgen kann und tritt die Möglichkeit einer solchen erst bei höherer Temperatur ein und zwar, wie aus folgenden Beobachtungen herrorgeht, am leichtesten wenn das Tetraoxybenzophenon in Lösung sich befindet. 
Beim Erhitzen des Ketons in trocknem Zustand auf $200-240^{\circ}$ wird 3,4 Dioxyxanthon*) gebildet, doch ist die Ausbeute keine gute. Erwärmt man es aber mit Wasser auf $180-220^{\circ}$, so erfolgt die Umwandlung fast quantitativ nach folgender Gleichung:

$$
\mathrm{C}_{6} \mathrm{H}_{5} \cdot \mathrm{OH}-\mathrm{CO} \cdot \mathrm{C}_{6} \mathrm{H}_{2}(\mathrm{OH})_{3}=\mathrm{C}_{0} \mathrm{H}_{4}<\underset{\mathrm{CO}}{\mathrm{O}}>\mathrm{C}_{6} \mathrm{H}_{2}(\mathrm{OH})_{2}+\mathrm{H}_{2} \mathrm{O} .
$$

Während Euxanthonsäure schon beim Kochen mit Ammoniak enthaltendem Wasser nach A. Baeyer's Beobachtung in Euxanthon übergeht, wird das $2,3,4,2$ Tetraoxybenzophenon erst beim Erhitzen mit verdünntem Ammoniak auf eine Temperatur von mindestens $150^{\circ}$ in das entsprechende Isoeuxanthon verwandelt. Bei niedriger Temperatur bilden sich bei Luftzutritt wesentlich Oxydationsproducte.

Aus der Thatsache, dass das hier besprochene Keton durch Wasserverlust in ein Xanthonderivat übergeht, haben wir schon früher die Schlussfolgerung gezogen, dass folgende Formel (I) anzunehmen sei.

*) Das 3, 4, Dioxyxanthon haben wir bisher keiner weiteren Untersuchung unterworfen. Wir theilen deshalb nur die analytischen Belege mit und verweisen, was die Eigensehaften betrifft, auf unsere frühere Mittheilung (Ber. d. deutsch. chem. Ges. 24, 969).

$0,3783 \mathrm{~g}$ krystallisirtes Dioxyxanthon verloren $0,0715 \quad \mathrm{H}_{2} \mathrm{O}$.

Berechnot für $3 \mathrm{H}_{2} \mathrm{O}$

19,16
Gefunden

19,32

$0,1645 \mathrm{gr}$ wasserfreies Dioxyxanthon gaben $0,4110 \mathrm{CO}$ und $0,0545 \quad \mathrm{H}_{2} \mathrm{O}$.

$\begin{array}{cc}\text { Berechnet } & \text { Gefunden } \\ 68,41 & 68,20 \\ 3,51 & 3,59\end{array}$

$0,1590 \mathrm{~g}$ Acetylderivat gaben $0,3835 \mathrm{CO}_{2}$ und $0,0535 \mathrm{H}_{3} \mathrm{O}$. $0,1610 \mathrm{~g} \quad " \quad 0,385 \overline{5} \mathrm{CO}_{2} \quad, \quad 0,0620 \mathrm{H}_{\mathrm{g}} \mathrm{O}$. Berechnet für Gefunden $\mathrm{C}_{13} \mathrm{H}_{6} \mathrm{O}_{4}\left(\mathrm{CH}_{3} \mathrm{CO}\right)_{2}$

H $3,94 \quad: 3,74 \quad 3,40$



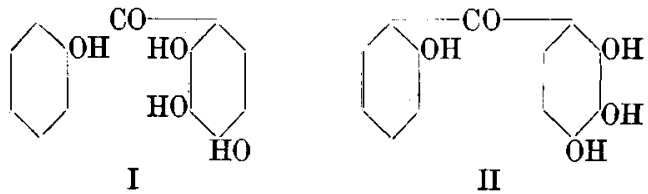

Dieselbe wird man wohl als identisch mit der Formel II ansehen dürfen, und es ist demnach der aus Salicylsäure und Pyrogallol entstehende Farbstoff als $2,3,4,2^{\prime}$ Tetraoxybenzophenon zu bezeichnen. Aus den obigen Beobachtungen darf man vielleicht den Schluss ziehen, dass die Lagerung der Atome bei niedriger Temperatur dem Schema II entspreche und erst beim Erwärmen eine solche wird, dass entsprechend Formel I die Wasserabspaltung möglich wird.

Durch Einwirkung von Brom auf das Keton bei Anwendung von Eisessig als Lösungsmittel erhält man in der Kälte ein Monobromtetraoxybenzophenon; beim Erwärmen dagegen Monobromdioxyxanthon und bei Ueberschuss von Brom ein Tribromderivat des Dioxyxanthons. Es geht hieraus hervor, dass durch den Eintritt von Brom die Xanthonbildung erleichtert wird,

Bromtetraoxybenzophenon.

Tetraoxybenzophenon wurde mit einer Lösung von Brom in Eisessig übergossen. Beim Schütteln trat Lösung ein und dann erstarrte diese plötzlich zu einem gelben Krystallbrei. Aus verdünntem Alkohol krystallisirt erhält man schwefelgelbe Prismen, welche bei $200^{\circ}$ schmelzen. In Soda oder stark verdünnter Kalilauge löst sich das Bromtetraoxybenzophenon mit gelber Farbe. Bei Ueberschuss von Kalihydrat bildet sich an der Luft ein grünes Oxydationsproduct, wodurch es sich von den Xanthonderivaten unterscheidet.

$0,1600 \mathrm{~g}$ gaben $0,0950 \mathrm{AgBr}$.

Berechnet für

Gefunden

$\mathrm{C}_{18} \mathrm{H}_{9} \mathrm{BrO}_{5}$

$\mathrm{Br}$

24,62

25,20 
Bromderivate des 3, 4 Dioxyxanthons.

Bromdiaxyxanthon, $\mathrm{C}_{13} \mathrm{II}_{7} \mathrm{BrO}_{4}$. Fügt man Brom zu einer warmen Iösung von Tetraoxybenzophenon in Eisessig und erhitzt einen Angenblick bis zum Sieden, so scheiden sich beim Erkalten röthliche Nädelchen aus. Durch Lösen in Soda, Fällen mit einer Säure und Krystallisiren aus Alkohol erhält man fast farblose Nadeln, welche bei $310^{\circ}$ sich zu bräunen beginnen, aber bei $360^{\circ}$ noch nicht geschmolzen sind. Ihr Verhalten gegen Alkalien zeigt, dass kein Derivat des Tetraoxybenzophenons mehr vorliegt. Sie lösen sich in Kalilauge mit blutrother Farbe, welche sich weder beim Stehenlassen noch beim Kochen ändert. Aus der Analyse geht hervor, dass ein Bromdioxyxanthon entstanden ist, dem wohl ein wenig eines bromreicheren Ierivats heigemengt war.

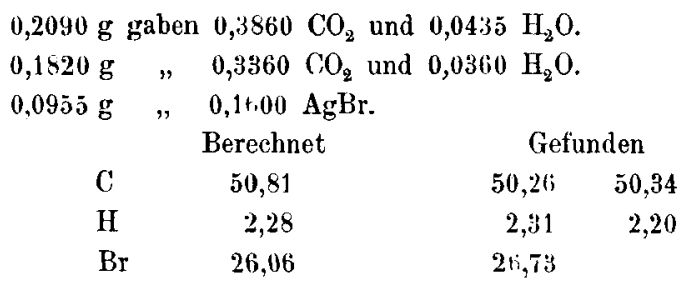

Durch concentrirtc Schwefelsäure wird es auch bei mehrstündigem Erwärmen auf $100^{\circ}$ nicht veründert.

Tribromdioxyxanthon, $\mathrm{C}_{13} \mathrm{H}_{5} \mathrm{Br}_{3} \mathrm{O}_{4}$. Giebt man zu der Lösung des Tetraoxybenzophenons in Eisessig einen grossen Ueberschuss von Brom und kocht längere Zcit, so entweicht reichlich Bromwasserstoff und man erhält nach dem Erkalten einen fast farblosen Körper, der bei $360^{\circ}$ noch nicht schmilzt und gegen Alkalien so beständig ist wie das Bromdioxyxanthon. Die Lösung in Kalilauge ist ticfroth und scheidet beim Stehen ein tiefrothes Salz aus.

Die Brombestimmung entspricht obiger Formel.

$$
0,1240 \mathrm{~g} \text { gaben } 0,1500 \mathrm{AgBr} \text {. }
$$

Berechnet

Gefunden

Br 


\section{Tetraoxynaphtylphenylketon.}

$$
\mathrm{C}_{6} \mathrm{I}_{2}(\mathrm{OII})_{3}-\mathrm{CO} . \mathrm{C}_{10} \mathrm{H}_{6} . \mathrm{OH} \text {. }
$$

Dieser Farbstoff entsteht aus Gallussäure und $\alpha$-Naphtol beim Erhitzen mit Chlorzink. Da nun allgemein bei diesen Ketonbildungen, wenn die Möglichkeit gegeben ist, das Carboxyl in Parastellung zum Hydroxyl tritt, so erscheint es uns am wahrscheinlichsten, dass dieses Tetraoxynaphtylphenylketon folgender Formel entsprechend zusammengesetzt ist:

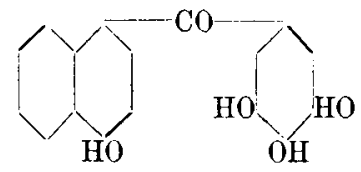

Mit Zugrundelegung des in der Einleitung aufgestellten Schemas wäre es daher als ein $4,3,^{\prime} 4,^{\prime} \check{\partial}^{\prime}$ Derivat zu bezeichnen. Beweise für die Richtigkeit dieser Auffassung baben wir bisher nicht auftinden können.

Das Tetraoxynaphtylphenylketon krystallisirt aus Alkohol in grüngelben Prismen oder Nadeln; es schmilzt nach vorhergegangener Braunfärbung bei $246^{\circ}$ (corr.). Es ist in Wasser, Benzol und Ligroïn unlöslich, löst sich kaum in Acther und ziemlich schwer in Alkohol und Eisessig. Mit concentrirter Schwefelsäure wird eine tiefrothe Lösung erhalten. In Alkalien löst es sich mit gelbrother Farbe.

Fs enthält 1 Mol. Krystallwasser, welches bei $100^{\circ}$ entweicht, wobei die Farbe der Substanz gelblicher wird.

$$
\begin{aligned}
& 0,3120 \mathrm{~g} \text { verloren bei } 115^{n} 0,0185 \mathrm{H}_{2} \mathrm{O} \text {. } \\
& \text { Berechnet für Gefunden } \\
& \mathrm{C}_{13} \mathrm{H}_{12} \mathrm{O}_{5}+\mathrm{H}_{2} \mathrm{O} \\
& \begin{array}{lll}
\mathrm{H}_{2} \mathrm{O} & 5,73 & 5,93
\end{array} \\
& \mathrm{C}_{13} \mathrm{H}_{12} \mathrm{O}_{5} \\
& \begin{array}{lll}
\mathrm{C} & 68,92 & 69,08
\end{array} \\
& \begin{array}{lll}
\mathrm{H} & 4,05 & 4,17
\end{array}
\end{aligned}
$$


Das Kcliumsalz, $\mathrm{C}_{17} \mathrm{H}_{11} \mathrm{O}_{5} \mathrm{~K}$, erhält man durch Eingiessen einer alkoholischen Kalilösung in eine alkoholische Lösung des Ketons als pulvrigen braunen Niederschlag.

$$
\begin{array}{ccc} 
& \begin{array}{c}
0,2080 \mathrm{~g} \text { gaben } \\
\text { Berechnet }
\end{array} & \begin{array}{c}
0540 \mathrm{~K}_{2} \mathrm{SO}_{4} . \\
\text { Gefunden }
\end{array} \\
\mathbf{h} & 11,68 & 11,66
\end{array}
$$

Lässt man umgekehrt die Ketonlösung in alkoholisches Kalihydrat fliessen, so entsteht keine Fällung, indem offenbar ein kaliumreicheres und löslicheres Salz sich bildet.

Das Natriumsalz, $\mathrm{C}_{17} \mathrm{H}_{11} \mathrm{O}_{5} \mathrm{Na}$, haben wir in Form schöner brauner Nadeln erhalten, durch Erwärmen des in Wasser suspendirten Ketons mit der berechneten Menge Natriumearbonat. Es ist in Wasser nur sehwicrig lüslich; bei längerem Kochen tritt theilweise Zersetzung ein.

$$
\begin{array}{ccc} 
& 0,2330 \mathrm{~g} \text { gaben } & 0,0520 \mathrm{Na}_{3} \mathrm{SO}_{4} . \\
& \text { Berechnet } & \text { Gefunden } \\
\mathrm{Na} & 7,23 & 7,14
\end{array}
$$

Hydrazon des Tetraoxynaphtylphenylketons.

Das Keton wurde mit Phenylhydrazin 8 Stunden lang in alkoholischer Lösung erwärmt. Beim Verdunsten des Alkohols schieden sich gelbbraune Blättehen aus, welche unter Zersetzung bei $210^{\circ}$ schmelzen. Sie sind in Alkohol, Aether und Eisessig löslich, dagegen in Benzol unlöslich. Die Analyse gab der Formel

$$
\begin{aligned}
& \mathrm{C}_{6} \mathrm{H}_{5}(\mathrm{OH})_{3} \\
& \mathrm{C}_{10} \mathrm{H}_{4}(\mathrm{OH})^{\prime}
\end{aligned}>\mathrm{C}^{\prime}=\text { N.NH. } \mathrm{C}_{6} \mathrm{H}_{5}
$$

entsprechende Zahlen.

$$
\begin{aligned}
& 0,1590 \mathrm{~g} \text { gaben } 0,4160 \mathrm{CO}_{\mathrm{a}} \text { und } 0,0730 \mathrm{H}_{2} \mathrm{O} \text {. } \\
& \text { Berechnet Gefunden } \\
& \text { C. } \quad \pi 1,50 \quad \pi 1,35 \\
& \mathrm{H} \quad 4,66 \quad 5,10
\end{aligned}
$$

Acetylderivat des Tetraoxynaphtylphenyllietons.

Das Keton wurde einige Stunden mit Essigsäureanhydrid gekocht und dann nur wenig Wasser zugesetzt. Es schieden 
sich nach und nach gelbe Krystalle aus, welche in Alkohol, Eisessig und Benzol leicht, in Aether schwer löslich sind und bei $129^{\circ}$ schmelzen.

Der Analyse nach enthalten sie vier Acetylgruppen.

$\begin{array}{ccc} & 0,1635 \mathrm{~g} \text { gaben } 0,3825 \mathrm{CO}_{2} \text { und } & \begin{array}{c}0,0660 \mathrm{H}_{2} \mathrm{O} . \\ \text { Grefunden }\end{array} \\ \text { Berechnet für } & \\ & \mathrm{C}_{12} \mathrm{H}_{8} \mathrm{O}_{5}\left(\mathrm{CH}_{3} \mathrm{CO}\right)_{4} & \\ \mathrm{C} & 64,66 & 63,80 \\ \mathrm{H} & 4,31 & 4,48\end{array}$

Verhalten gegen Schwefelsäure und Wasser.

Um vielleicht das Keton in Pyrogallol und eine Oxynaphtoësäure zu spalten und so $\mathrm{zu}$ eincm bestimmten Beweis für die Richtigkeit obiger aufgelösten Formel zu kommen, haben wir das Tetraoxynaphtylphenylketon mit Schwefelsäure von verschiedener Concentration behandelt, aber als Reactionsproducte nur Gallussäure und Naphtol erhalten.

Wird das Keton mit concentrirter Schwefelsäure eine halbe Stunde auf $100^{\circ}$ erwärmt, so geht die blutrothe Farbe allmälig in eine dunkelrothe über und es macht sich ein schwacher Naphtolgeruch bemerkbar. Schüttelt man nach dem Verdünnen durch Wasser mit Aether aus, so erhält man geringe Mengen eines schwefelfreien, bci $240^{\circ}$ schmelzenden Körpers. Dasselbe Resultat wurde beim Erwärmen des Ketons mit Schwefelsäure, welche mit dem gleichen Volum Wasser verdünnt war, auf $140^{\circ}$ erhalten. Am besten verlief die Spaltung, als wir $0,5 \mathrm{~g}$ Keton mit $5 \mathrm{~cm} \mathrm{II}_{2} \mathrm{SO}_{4}$ und $2,5 \mathrm{ccm} \mathrm{H}_{2} \mathrm{O}$ so lange auf $130^{\circ}$ erhitzten, bis Dunkelfärbung eintrat, was nach ungeführ einer halben Stunde der Fall war. Wurde dic Reaction in einer längeren, mit einem Glasstopfen verschlossenen Röhre vorgenommen, so setzten sich an letzterem Krystalle von Naphtol $\mathrm{ab}$, welche den richtigen Schmelzpunkt $96^{\circ}$ zeigten. Es wurde mit Wasser verdünnt, von einer geringen Menge unveränderter Substanz abfiltrirt und mit Aether ausgeschüttelt, welcher beim Verdunsten schwach gelblich gefärbte Nädelchen hinterliess, die 
constant bei $246^{\prime \prime}$ (corr.) schmolzen und den Eigenschaften und der Analyse nach nur aus Gallussäure bestanden.

$\begin{array}{cccc} & 0,1565 \mathrm{~g} \text { gaben } & 0,2555 \mathrm{CO} \mathrm{CO}_{2} \text { und } \begin{array}{c}0,0600 \mathrm{H}_{2} \mathrm{O} . \\ \text { Gefunden }\end{array} \\ \mathrm{C} & \text { Berechnet } & 44,52 \\ \mathrm{H} & 44,69 & 4,25\end{array}$

Bei Versuchen mit verdünnter Schwefelsäure und bei höherer Temperatur trat entweder Verkohlung ein oder das Keton blieb unverändert. Auch beim Erhitzen mit Wasser auf $200^{\circ} \mathrm{er}-$ folgte keine Veränderung; wurde dic Temperatur bis auf $225^{0}$ gesteigert, so trat Zersetzung ein, und in Folge von Kohlensäureabspaltung war starker Druck in der Röhre. Aus dem erhaltenen Product liess sich leicht Naphtol isoliren; cin xanthonartiges Derivat hatte sich nicht gebildet, was auch bei der Stellung der Iydroxyle ohne Umlagerung nicht möglich ist.

Figenthümlich verläuft die Finwirkung von Brom auf das Keton; es wurden an Stello eines Substitutionsproductes bromhaltige Körper erhalten, wclche sanerstoffärmer, wohl als Aether anzusehen sind, dic sich von zwei Molekülen tribromirten Ketons weniger ein Molekül Wasser ableiten.

Zu dem in Eisessig suspendirten Tetraoxynaphtylphenylketon wurde cin Uebersehuss ciner Lösung von Brom in Eisessig zugegeben und tüchtig geschüttelt, wobei das Keton in Lösung geht, dann scheidet sich plötzlich cin gelb gefärbter Körper aus, der abgesaugt und mit verdünnter Essigsäure ausgewasehen wurde. Aus Alkohol umkrystallisirt schmolz er bei $293^{\circ}$ (corr.); er löst sich ziemlich leicht in Alkohol, Eisessig und Aether, aber nur spurenweise in Ligroïn und Benzol.

$\begin{array}{lccc} & 0,1695 \mathrm{~g} \text { gaben } 0,2440 \mathrm{CO} \text {, und } 0,0295 \mathrm{H}_{2} \mathrm{O} \text {. } \\ & 0,1295 \mathrm{~g}, \quad 0,1410 \mathrm{AgBr} . & \\ & \text { Berechnet für } & \text { Gefunden } \\ & \mathrm{C}_{34} \mathrm{H}_{40} \mathrm{O}_{9} \mathrm{Br}_{\mathrm{C}} & \\ \mathrm{C} & \mathbf{3} 9,00 & 39,25 \\ \mathrm{H} & 1,53 & 1,93 \\ \mathrm{Br} & 45,80 & 46,34\end{array}$


Graebe und Eichengrün, Oxyketonfarbstoffe. $\quad 317$

Eine andere bei $246^{\circ}$ schmelzende Verbindung wurde erhalten, als mit Eisessig vermischtes Brom so lange zu einer alkoholischen Lösung des Ketons gegeben wurde, bis die Farbe des Broms nicht mehr verschwand. Beim Erkalten der stark erwärmten Flüssigkeit schieden sich gelbe prismatische Krystalle aus, die in Alkohol und Eisessig ausserordentlich schwer löslich sind.

$0,2030 \mathrm{~g}$ gaben $0,2960 \mathrm{~g} \mathrm{CO}_{3}$ und $0,0220 \mathrm{H}_{2} \mathrm{O}$ entsprechend $39,81 \mathrm{C}$ und $1,25 \mathrm{H}$.

Dem Kohlenstoff - und Wasserstoffgehalt nach könnte eine noch sauerstoffärmere Verbindung, $\mathrm{C}_{17} \mathrm{H}_{7} \mathrm{O}_{4} \mathrm{Br}_{3}$, vorliegen, welche 39,61 pC. C und 1,36 H verlangt.

Concentrirte Salpetersäure reagirt bei mässigem Erwärmen äusserst heftig mit dem Tetraoxynaphtylphenylketon und bewirkt vollständige Oxydation. Durch verdünnte Salpetersäure erhält man beim Erwärmen auf $40-50^{\circ}$ einen Körper, der aus Alkohol in gelben Nadeln krystallisirt und bei $134^{0}$ unter Zersetzung schmilzt. Er löst sich in Kalilauge, doch scheiden sich nach kurzer Zeit braune Nadeln, das Kaliumsalz, aus.

Die Analyse gab Zahlen, welche einem Dinitroderivat entsprechen.

$\begin{array}{ccc} & 0,1510 \text { g gaben } 0,2935 \mathrm{CO}_{2} \text { und } & 0,0320 \mathrm{H}_{2} \mathrm{O} . \\ \text { Gerechnet für } & \text { Gefunden } \\ & \mathrm{C}_{17} \mathrm{H}_{10} \mathrm{O}_{5}\left(\mathrm{NO}_{2}\right)_{3} & \\ \mathrm{C} & 53,40 & 53,00 \\ \mathrm{H} & 2,62 & 2,72\end{array}$

